\title{
Keratan Sulfate Proteoglycans in Plasticity and Recovery after Spinal Cord Injury
}

\author{
Brett J. Hilton, ${ }^{1}$ Bradley T. Lang, ${ }^{2}$ and Jared M. Cregg ${ }^{2}$ \\ ${ }^{1}$ Department of Zoology and International Collaboration on Repair Discoveries, The University of British Columbia, Vancouver, British Columbia, Canada \\ V5Z 1M9 and 2Department of Neurosciences, Case Western Reserve University, Cleveland, Ohio 44106 \\ Review of Imagama et al.
}

The limited potential for regeneration and plasticity of adult mammalian CNS axons is a major impediment to functional recovery following spinal cord injury (SCI). Inhibitory extracellular matrix, particularly chondroitin sulfate proteoglycans (CSPGs), associated with the lesion penumbra and astroglial scar are a major cause of regeneration failure. CSPGs are a diverse class of macromolecules consisting of a core protein posttranslationally modified with chains of chondroitin sulfate (CS) glycosaminoglycan. One successful approach to enhancing plasticity and promoting functional recovery has been CS degradation using the enzyme chondroitinase ABC (chABC) (Bradbury et al., 2002). Keratan sulfate proteoglycans (KSPGs) are another class of inhibitory extracellular matrix molecule that are associated with spinal cord lesions (Snow et al., 1990; Jones and Tuszynski, 2002). Mice lacking GlcNAc6ST-1, an enzyme critical for keratan sulfate (KS) biosynthesis, have enhanced plasticity and functional recovery after SCI (Ito et al., 2010),

Received Jan. 22, 2012; revised Feb. 14, 2012; accepted Feb. 16, 2012.

This work was supported by the National Science Foundation Graduate Research Fellowship Grant DGE-0951783 to J.M.C. We thank the 14th International Symposium on Neural Regeneration for facilitating this collaboration. We thank Drs. Wolfram Tetzlaff and Jerry Silver for their supervision and support.

Correspondence should be addressed to Brett J. Hilton, International Collaboration on Repair Discoveries, Blusson Spinal Cord Centre, 818 West 10th Avenue, Vancouver, British Columbia, Canada, V5Z 1M9. E-mail: bhilton@icord.org.

DOI:10.1523/JNEUROSCI.0333-12.2012

Copyright $\odot 2012$ the authors $\quad 0270-6474 / 12 / 324331-03 \$ 15.00 / 0$ but therapeutic agents targeting KSPGs have not been explored.

In a recent study, Imagama et al. (2011) assessed the ability of a KS-specific degradative enzyme, keratanase II (K-II), to promote axonal plasticity and functional recovery independent of and in tandem with chABC in a rat contusion model of acute SCI. The authors purified K-II from $B$. circulans until it showed no chondroitinase activity (Imagama et al., 2011, their Table 2). This step was critical in resolving distinct roles for both CSPGs and KSPGs in axonal inhibition. Osmotic minipumps delivered each therapeutic regimen intrathecally for $14 \mathrm{~d}$, consistent with the time at which post-SCI glycosaminoglycan levels are highest (Imagama et al., 2011, their Fig. 1A,B).

To determine whether K-II treatment enables recovery of hindlimb motor function after SCI, the authors used the Basso, Beattie, and Bresnahan (BBB) locomotor rating scale, a 22-point scale where 0 represents no hindlimb movement and 21 represents normal locomotion. The improvements in motor recovery following K-II treatment were striking. By 4 weeks postinjury, vehicle-treated rats had an average score of $\sim 6$, representing movement in three hindlimb joints (Imagama et al., 2011, their Fig. 2A). In contrast, K-II-treated rats achieved an average score of $\sim 13$, corresponding to weightsupported plantar-stepping and frequent forelimb-hindlimb coordination. These differences persisted up to $70 \mathrm{~d}$ postinjury with weekly assessment, long after the 2 week treatment period with K-II was ended. Thus, K-II treatment promotes substantial motor recovery following SCI. chABC-treated rats achieved a score of $\sim 11$, pointing to a similar functional effect of CS and KS (Imagama et al., 2011, their Fig. 2A). Interestingly, treatment with both chABC and K-II treatment had no synergistic or additive effect on locomotor recovery compared with treatment with each enzyme alone (Imagama et al., 2011, their Fig. 2F). This comes as a surprise, as each enzyme targets molecularly distinct glycosaminoglycan moieties, and suggests that CS and KS may act through the same mechanism to impede functional recovery.

It is unclear why vehicle-treated animals had an average BBB score of $\sim 6$, which is considerably lower than in similar studies, in which scores averaged $\sim 10$ using the same impact force at the same spinal cord level (Scheff et al., 2003). Although not specifically addressed, the implantation of a catheter into the subarachnoid space may result in further compression damage to the cord (Jones and Tuszynski, 2001). All animals received intrathecal catheters regardless of treatment. Nevertheless, the low BBB score for vehicle-treated animals makes it more difficult to interpret the behavioral outcomes following treatment. It is possible that the differences would not persist if rats exhibited the extent of spontaneous recovery that normally occurs in this 
model of contusion injury, and it cannot be ruled out that recovery of the animals following enzyme treatment was due to alleviation of catheter-induced damage.

Histological assessments suggest that axonal plasticity is one mechanism underlying functional recovery in K-II-treated rats. Disruption of serotonergic and corticospinal fibers contributes to paralysis following SCI, and counts of 5HT-positive fibers indicated that K-II treatment promoted serotonergic fiber sprouting caudal to the injury site (Imagama et al., 2011, their Fig. $4 B$ ), whereas anterograde tracing of the corticospinal tract indicated modest corticospinal collateral sprouting in gray matter caudal to the lesion site, with reconstitution to $<12 \%$ of that seen in sham injured animals (Imagama et al., 2011, their Fig. 4D). Therefore, removal of keratan sugars from the spinal cord milieu increases plasticity and sprouting. Such local sprouting can promote recovery by bypassing the lesion site through relay connections with propriospinal interneurons (Vavrek et al., 2006), but with increased branching observed so far caudally to the lesion site, it is possible that direct descending input to spinal motor neurons was reestablished in this case (Fig. 1). chABC treatment showed a similar impact on fiber counts to K-II treatment, but no histological assessment was made of K-II and chABC cotreated animals. This analysis would be helpful in ascertaining the relationship between CS and KS on restricting plasticity following SCI.

To further test the effects of K-II and chABC on sprouting, Imagama et al. (2011) subjected cultured cerebellar granule neurons to either aggrecan $(5 \mu \mathrm{g} / \mathrm{ml})$ or aggrecan that had been predigested with chABC, K-II, or both. Aggrecan core protein is unique in that it is modified with both CS and KS chains. The authors found that enzymatic removal of either CS or KS chains resulted in increased neurite length and, similar to their behavioral data, chABC/K-II did not act synergistically (Imagama et al., 2011, their Fig. 6C). These results indicate that both CS and KS chains are necessary for aggrecan to exert inhibition, suggesting that aggrecan signals as a whole molecule. However, a previous report showed a synergistic effect on neurite outgrowth following keratanase and chABC treatment, although these studies used a mixture of CSPGs and KSPGs instead of a single molecule with both modifications (Snow et al., 1990). Conceivably, aggrecan-mediated neurite inhibition could require both CS and KS integrity, but with a variety of CSPGs and KSPGs present following SCI, further as-

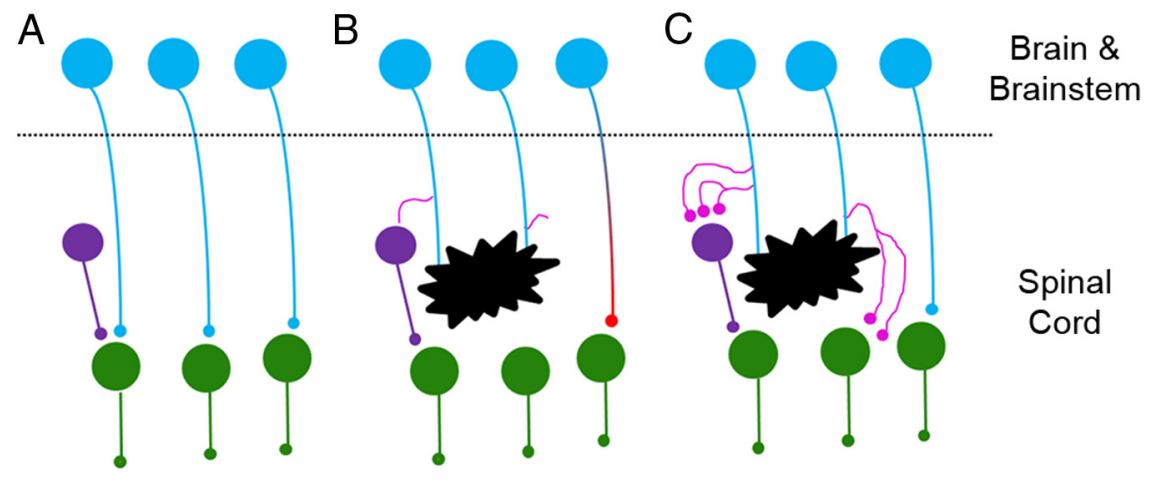

Figure 1. A, Descending supraspinal tracts (corticospinal, rubrospinal, etc.; blue) synapse on premotor interneurons and

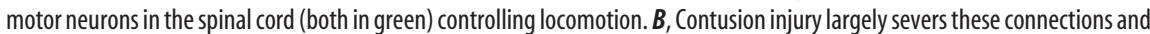
disrupts axonal conduction in spared fibers (red). Limited plasticity (pink) may allow some restoration of function through propriospinal neurons (purple), which can form relay circuits. C, Blocking the inhibitory action of KSPGs with K-II treatment could enhance this plasticity or promote branching past the lesion to reestablish direct supraspinal input. Additionally, K-II might also promote conduction in spared fibers.

sessment of the relationship between the two glycosaminoglycans is warranted. Moreover, our understanding of the molecular machinery underlying proteoglycan restriction of plasticity is incomplete. One natural hypothesis for the lack of synergy between KSPGs and CSPGs found by Imagama et al. (2011) is a shared common receptor or pathway underlying neurite outgrowth. For example, KSPGs might also signal with CSPGs and HSPGs through receptor protein tyrosine phosphatase- $\sigma$ (Coles et al., 2011). Combined treatment could thus target the same receptors and signaling pathways as treatment with chABC or K-II alone, leading to a ceiling effect on plasticity.

Other mechanisms for K-II-mediated functional recovery could also be at work. chABC treatment can exert neuroprotective and trophic effects; the extent to which K-II acts similarly remains unknown. Additionally, recent work by James et al. (2011) identifies disruption of conduction in spared axons following contusive $\mathrm{SCI}$ as an alternative means by which functional recovery might be impeded, even into the chronic stages of injury. CS and KS inhibition of functional recovery might occur, in part, through the same process (Fig. 1). Conduction blockade by CSPGs has been observed both in a lateral hemisection model of SCI and following purified CSPG injection into the lateral columns of the uninjured cord, albeit through unknown mechanisms (Hunanyan et al., 2010). James et al. (2011) surmise that disruptions in conduction might result from a lack of remyelination of axons that are denuded by the initial trauma. Indeed, CSPGs have been shown to inhibit process outgrowth and differentiation of oligodendrocyte precursors in vitro (Siebert and Osterhout, 2011). Notably, electro- physiological assessment of motor-evoked potentials and spinal cord-evoked potentials 8 weeks postinjury indicated that chABC and K-II treatment both ameliorated the impact of contusion on mean latency, corresponding to a conduction velocity rescue effect (Imagama et al., 2011, their Figs. 2C, 3C). Future characterization of the effects of CSPGs and KSPGs on axonal conduction and remyelination may provide further insights into their roles in spinal cord repair.

In summary, Imagama et al. (2011) demonstrate that KSPGs represent a major molecular inhibitor of plasticity after injury. Acute K-II treatment degraded KSPGs and allowed substantial motor recovery. It will be informative to undertake future experiments that determine whether K-II promotes recovery when administered in subacute or chronic stages of SCI, as these are more clinically relevant time points in which K-II treatment could conceivably be initiated in humans.

\section{References}

Bradbury EJ, Moon LD, Popat RJ, King VR, Bennett GS, Patel PN, Fawcett JW, McMahon SB (2002) Chondrotinase ABC promotes functional recovery after spinal cord injury. $\mathrm{Na}$ ture 416:636-640.

Coles CH, Shen Y, Tenney AP, Siebold C, Sutton GC, Lu W, Gallagher JT, Jones EY, Flanagan JG, Aricescu AR (2011) Proteoglycan-specific molecular switch for RPTP $\sigma$ clustering and neuronal extension. Science 332:484-488.

Hunanyan AS, García-Alías G, Alessi V, Levine JM, Fawcett JW, Mendell LM, Arvanian VL (2010) Role of chondroitin sulfate proteoglycans in axonal conduction in mammalian spinal cord. J Neurosci 30:7761-7769.

Imagama S, Sakamoto K, Tauchi R, Shinjo R, Ohgomori T, Ito Z, Zhang H, Nishida Y, Asami N, Takeshita S, Sugiura N, Watanabe $H$, 
Yamashita T, Ishiguro N, Matsuyama Y, Kadomatsu K (2011) Keratan sulfate restricts neural plasticity after spinal cord injury. J Neurosci 31:17091-17102.

Ito Z, Sakamoto K, Imagama S, Matsuyama Y, Zhang H, Hirano K, Ando K, Yamashita T, Ishiguro N, Kadomatsu K (2010) Nacetylglucosamine 6-O-sulfotransferase-1deficient mice show better functional recovery after spinal cord injury. J Neurosci 30:5937-5947.

James ND, Bartus K, Grist J, Bennett DL, McMahon SB, Bradbury EJ (2011) Conduction failure following spinal cord injury: functional and anatomical changes from acute to chronic stages. J Neurosci 31:18543-18555.

Jones LL, Tuszynski MH (2001) Chronic intrathecal infusions after spinal cord injury cause scarring and compression. Microsc Res Tech 54:317-324.

Jones LL, Tuszynski MH (2002) Spinal cord injury elicits expression of keratan sulfate proteoglycans by macrophages, reactive microglia, and oligodendrocyte progenitors. J Neurosci 22: 4611-4624.

Scheff SW, Rabchevsky AG, Fugaccia I, Main JA, Lumpp JE Jr (2003) Experimental modeling of spinal cord injury: characterization of a force-defined injury device. J Neurotrauma 20:179-193.

Siebert JR, Osterhout DJ (2011) The inhibitory effects of chondroitin sulfate proteoglycans on oligodendrocytes. J Neurochem 119:176-188.

Snow DM, Lemmon V, Carrino DA, Caplan AI, Silver J (1990) Sulfated proteoglycans in astroglial barriers inhibit neurite outgrowth in vitro. Exp Neurol 109:111-130.

Vavrek R, Girgis J, Tetzlaff W, Hiebert GW, Fouad K (2006) BDNF promotes connections of corticospinal neurons onto spared descending interneurons in spinal cord injured rats. Brain 129:1534-1545. 